A new epoch in surgery will dawn when surgeons realize, 9.s I have done, that they can leave quite a large amount of infection to be dealt with by the inherent resistance of the body to infection.

These notes are written with the sole object of stimulating my seniors and contemporaries who have more material to carry out the lines I have suggested, and to more and more practise primary suture in their cases, and above all to give up once and for all the use of open rigid drainage tubes.

Sir Bertrand Dawson, G.C.V.O., has kindly added the following note on the treatment of streptococcal empyemata:

With regard to empyemata, my experience is that the pneumococcal ones do well with immediate closure after evacuation, but not the streptococcal. These latter need to be drained for two or three days, with or without Carrel tubes, and then they can be sewn up.

I agree that streptococcal infections must be dealt with on different lines from staphylococcal, pneumococcal, and other infections, but in civil practice it is very rare to meet with streptococcal empyema.

\section{ANOMALOUS MUSCULAR ACTION IN NERVE INJURIES.}

By D. M. HUGHES, M.B., F.R.C.S., MaJor R.A.M.C., OFFICER IN CHARGE OF SURGICAL DIVIBION, EDMONTON MILITARY HOBPITAL.

THE precise action of an individual muscle is not always easy to determine in the uninjured limb, since a particular movement is in most cases brought about by the resultant action of many muscles and not by one alone. When a nerve trunk is severed and the muscles supplied by it are put out of action, the muscles supplied by the uninjured nerves of the same region sometimes give rise to surprising movements. It is probable, in fact, that the movement resulting in these cases is such as never occurs in a limb with properly balanced muscles. While this movement is in many cases of mere academic interest, it occasionally happens that it may flatter the surgeon who has performed a nerve suture, giving him the impression that muscular recovery has begun, whereas an attentive examination of the muscles causing the movement may show that his conclusion is premature.

One of the most striking instances of this that have come under my notice is that when the median and ulnar nerves are divided in the npper arm, active flexion of the wrist is often still present, being brought about by the extensor ossis metacarpi pollicis. Another anomaly is seen in posterior interosseous paralysis, namely, the first Jorsal internsseous muscle can cause a lifting of the thumb easily confused with commencing recovery of extension of that digit. These two anomalous movements clearly depend upon the difference of the plane in which the metacarpal bone of the thumb lies relatively to the plane of the four remaining metacarpals.

Other unexpected actions are that the terminal phalanx of the thumb can be actively extended in cases of posterior interosseous paralysis, the extension of course being brought about by the insertion of the abductor pollicis into the dorsal expansion of the extensor tendon; and that in nlnar paralysis the index finger can be slightly abducted by the obliquity of the extensor tendons passing to that digit. In persistent paralysis these movements, aided by use, massage, and électrical stimulation, may acquire some importance.

Some of these unsuspected muscle actions, it is true, may indicate merely that one's memory for anatomical letail requires furbishing, and anatomists could doubtless furnish nuany more instances. 'There is no doubt, however, that a partially paralysed limb offers a rich field for the investigation of the essential action of individual muscles.

Another anomaly which may often be observed is due to the general preponderance of the flexor muscles. This is seen in its most marked form in lesions of the upper motor neuron-for example, in a case of hemiplegia. If the wrist of such a patient is passively extended, the fingers curl into the palm and exercise a quite perceptible grip. In a lower motor neuron paralysis-for example, in complete lesion of the median and ulnar nerves in the upper arm-this action is also observable, though it is not so evident. and may give rise to a false notion that recovery of active flexion of the fingers is in progress. In some cases it certainly indicates that the elas ticity, and therefore the nutrition, of the finger-flexors is being maintained, but in others it points to essential fibrosis and shortening of those muscles, and is consequently of grave significance. In the latter case it usually betrays neglect of treatment. If the fingers can be straightened only while the wrist is flexed this condition of fibrosis is present in the flexor: of the fingers. In a most helpful paper Professor Langley. ${ }^{1}$ has shown that the danger of stretching a paralysed muscle has been somewhat overstated. It is questionable, at any rate, whether it is preferable to risk stretching the muscles or to encounter a case of musculo. spiral paralysis so assiduously treated on a "cock-up" splint that the wrist, by reason of fibrosis of the extensors, could be brought neither up nor down.

It is surprising, again, to find what little muscular disability may result from complete division of the median nerve at the wrist. In a case recently operated upon there was, except for most intractable trophic ulcers over the median distribution in the hand, which was completely anaesthetic, practically no disability, and the lesion mighit easily have been overlooked. The abductor pollicis was completely paralysed and its situation represented by a fossa on the radial side of the metacarpal bone, but the loss of this muscle seemed not to affect the utility of the thumb. As for the movement of opposition, this was quite efficiently carried out by the adductores pollicis in: combination with the long and short flexors, both heads of the latter appearing to be active in spite of the complet $\epsilon$ division of the nerve an inch above the wrist. It is interesting to note that within a month the troplic ulcer: healed for the first time, but there was still complete proto. pathic loss. This may only indicate, however, that the hand had received better protection after the operation than it had previously obtained.

Cases such as this are of interest from the ease with which nerve lesions may be overlooked, as pointed out by Mr. T. E. Hammond ${ }^{2}$ in the case of the internal popliteal. 'They also illustrate the point that if a nerve is sutured without a previous careful examination of both muscular and sensory loss, the operator may claim results to which he is not entitled. This may account for some cases of atypically early recovery after nerve suture.

$$
\text { REFFrences. }
$$

${ }^{1}$ Langley: The effect of intermittent stretching on muscles and nerves after suture, Brirish MEDICAL JovRNAL, February 2nd. 1918. 2 Hammond: The involvement of the external and internal popliteal nerves in lesions of the sciatic nerve, BRITISH MEDICAL JOCRNAL, April 6th, 1918.

\section{EARIY CARDIAC BREATHLESSNESS AND “BUFFER-SAL'I” DEFICIENCY IN THE BLOOD.}

By BEN.JAMIN MOORE, D.Sc., F.R.S., DEPARTMENT OF APPLIED PHYSIOLOGY, MEDICAL RESEARCH COMMITTEE.

The review in the Journal of June 22nd (pp. 702-4) of the report of Dr. Lewis dealing with the causation of breath. lessness on exertion in early cardiac cases is most interest. ing, but may I be allowed to point out that the expression "buffer salts," first coined in Germany for an effèct described and measured originally in Britain, conveys an erroneous impression, because the protective action against variations in acidity or allalinity is due not to the inorganic salts, carbonates, or phosphates, of the plasma, but almost in its entirety to the amphoteric proteins?

This protective action was fully described, and a quanti. tative method supplied for its estimation, by the writer and his colleagues Roaf, Wilson, and Whitley at the biochemical laboratory of the University of Liverpool over a decade ago, the results being published in the Proceedings of the Royal Society and the Bio-chemical Journal. ${ }^{1}$

The action was described under the title of the "reactivity of the serum," as distinct from the "reaction of the serum" at any given moment, and by estimating the amount of decinormal alkali and acid necessary to titrate from one definite value of hydrogen ion concentration to another, marked by the mid-point or neutral point to two coloured indicators, namely, phenolphthalein and methyl orange, there was assigned a quantitative value to the "reactivity" or protective power of the blood and tissue 
fluids. This had to pass to the Continent and be re-dubbed the "buffer-salt effect" before receiving attention, and even now these earlier papers seem to be unknown to ljritish workers on the subject, although their results would well repay a little consideration.

For example, the expression "buffer-salt" is quite misleading; the effect is not due to salts, for the very sufficient reason that there does not exist a sufficient quantity of sodium carbonates and phosphates in the plasma in toto to account for one-tenth of the protective influence, or to carry one-quarter of the carbon dioxide found in arterial blood. Salts cannot protect which are not there, and at least 90 per cent. of the protective action is due to the plasma proteins.

A most important point brought out by this earlier work which appears to havo been quite forgotten is that all the inorganic salts of the plasma, including even the sodium chloride, are held in union by the proteins. It is this union which regulates the amount of salt in the blood with such a delicate precision. The excretory cells allow no excess of free salt in the circulating plasma, and it is this combining power of the proteins for salts which determines the value of physiologically " normal saline."

If the molecular concentration of mainmalian serum be determined from the depression of its freezing point it will be found to be approximately equivalent to that of a one-sixth molecular solution of sodium chloride. If, now, the same serum be titrated with standard acid till neutral to an indicator such as methyl orange, and then with standard alkali till neutral to phenolphthalein, the range will again be found equivalent to a one-sixth inolecular solution. Outside these two marginal limits very small additions of acid or alkali swing up or down the hydrogen ion concentration with enormous speed; within the limits large additions of acid or alkali produce only inoderate effects.

Within these viable limits, marked out by the titratable value of the proteins, variations by accumulation of organic acids and alkali and carbon dioxide occur in varying conditions of health and disease, and the neuro-muscular and cardiac system may be chronically fatigued by such products. The soldiers investigated by Dr. Lewis may owe their condition to the strain of neuro-muscular fatigue in duced by a physical training for which they are unfitted by nature. Apart from military training, the prolonged strain of war conditions is producing such an action on many people in civil life. Breathlessness, accompanied by disordered heart rhythm, is a prominent symptom, especially after any prolonged neuro-muscular exertion, in many persons bordering upon neurasthenia at the present time.

A variation in the balance of "reactivity" of the blood may also be present in the different forms of "shock." It lias been shown by Roaf and Adamson that the serum proteins completely lose their osmotic pressure when acid is added to the serum till the equi-potential point or point of physico-chemical neutrality is reached. Bayliss has recently shown that colloids such as gum acacia or gelatine, which were demonstrated by the earlier work of Moore and Roaf to have a high osmotic pressure, possess the property of maintaining for much longer periods than purely saline solutions a higher blood pressure under shock conditions.

These and kindred problems are intimately connected with that protective mechanism in the blood plasma first described and measured in Britain under the term of "reactivity of the serum," and later reintroduced from Grermany under the name of "Puffer-salzen" or. "buffer effect."

REFERENCE

1 Moore, Roaf and Whitley Proc, Roy Soc, B, vol 77, p. 102 (1905) and Moore and Wilson, Biochem. Journ., vol. i, p. 297 (1906).

\section{CARDIOPTOSIS.}

BY

W. GORDON, M.D., F.R.C.P.,

PHYSICIAN, DEVON AND EXETER HOSPITAL.

I "AM not sure whether the term "cardioptosis" has yet found its way into cardiology. I do not remember coming across it, and it is not in any of my textbooks. Yet $I$ am sure the term would serve a useful purpose in focussing attention on a condition not unlikely (as in one instance here related) to be misinterpreted. The condition, of which I can only speak from the clinical standpoint and from my limited experience, would appear to be one of undue laxity of the cardiac supports, such that, whilst the apex beat in recumbency is found about the normal posi tion, in the fifth space, in the erect position it is located just below this, in the sixth space, without any evidence of other causes to account for the displacement.

In the first of the two following cases there was no sign of enlargement of the heart, although " enlargerl heart" had been diagnosed, partly from the position of the apex beat in the sixth space; and in this case the dropped heart was associated with an obviously dropped kidney and a probably dropped stomach. In the second case there was moderate cardiac enlarge. ment, although not so great as to seem to account for such a displacement of the apex beat in the erect position; a palpable spleen with normal temperature and no obvious blood changes in a fresh film, suggested in this second case a dropped spleen, but this could not be certainly diagnosed in the limited time at my disposal.

\section{CASE I.}

M. E., aged 21, suffered from attacks of "faintness" not however, going to complete unconsciousness. He had had diphtheria badly at $1 \frac{1}{2}$ years old, followed by "paralysis of hands and feet," and could not walk till 3 . He had had double pneumonia at 11 and a bad fall at 17 ; he had also been physically overworked, I think.

The chest was slightly deformed, the third and fourth rib cartilages on both sides being unduly prominent at their sternal junctions. Urine, lungs, and reflexes were normal. There was marked nephroptosis on the right side, and the lower there was the stomach (which seemed, however, to be somewhat dilated was close to the umbilicus. however, to be somewhat dilated) was close to the umbilicus. He was supposed to have "enlarged heart," but my examination gave the following results : In sternal line only $\frac{1}{2}$ in. rightwards and $3 \frac{1}{4}$ in. leftwards. The apex beat was quite obviously in the sixth left space, 3 in. from the mid-line of the sternum. In the recumbent position the right margin of dullness was at the mid-sternum and reached leftwards 3 in. The apex beat in recumbency was obviously in the fifth space at about the edge of the dullness. There was no the fifth space at about the edge of the dullness. There was no
murmur in the recumbent position, but in the erect position a very local short systolic murmur was heard 1 in. outside the apex beat during inspiration only, being completely lost during sustained expiration.

\section{Case II.}

J. S., 48, complained of weakness and occasional shortness of breath, had considerable rheumatoid arthritis, and was more or less a chronic invalid.

In the erect position the cardiac dullness reached rightwards to mid-sternum and leftwards to the left mammary line, 4 in. from mid-sternum; upwards to the fourth rib. The apex beat was unmistakably in the sixth space. In the r. bent position the dullness was, as usual, narrower but reached as high as the third rib. The apex beat was definitely in the fifth space inside the left mammary line.

There was a systolic murmur, heard in both positions, louder after exertion and unaffected by respiration, where the fifth rib cartilage joined the sternum. Urine, lungs, and reflexes were normal. In the abdomen the splenic edge descended on inspira. tion about a fingerbreadth below the left ribs. The blood in fresh film seemed normal and the temperature was normal.

\section{fftemoranda:}

\section{MEDICAL, SURGICAL, OBSTETRICAL.}

\section{THRESHOLD TESTS.}

MUCH is to be learnt by investigating the thresholds for light and colour. Finding by routine examination that a normal standard, quite as uniform as that for visual acuity, exists, I have recently attempted to standardize a series of test objects which can be universally used for determining how near to, or how far from, the normal standard each individual eye is. In diseases of the retina, the choroid, and the optic nerve, very remarkable variations from the normal are found. But I have reason to believe that ab. normal thresholds are not limited to diseases of the eye. marked changes in the thresholds are certainly to be found where the ocular implication is secondary. Thus working with my test, my colleague, Dr. Walter Sinclair of Ipswich, pointed out that in several of his cases of retinitis of pregnancy the threshold for yellow was the only one attacked, but that very considerably. I found the same in the only case I have seen since. The threshold for yellow was reduced to recognition of the dilution 1 in 4 , instead of the normal 1 in 32 .

Though this can be no more than a bypothesis, I suggest that those who are not limited, like myself; to ophthalmic 